\title{
Functional-Foods Use: A Novel Dietary Regimen for Type 2 Diabetes Treatment and Management
}

\author{
A.S. OLAGUNJU ${ }^{1 *}$,F.G. OLORUNFEMI ${ }^{2}$, O.J. TEIBO ${ }^{1}$, S.A BELLO ${ }^{1}$, T.E FABUNMI ${ }^{4}$, O.M AJAO ${ }^{3}$, \\ 1. Department of Biochemistry, Faculty of Basic Medical Sciences, University of Ibadan, Ibadan, Nigeria. \\ 2. Department of Biochemistry, College of medicine, University of Lagos, Nigeria. \\ 3. Department of Biochemistry, Modibbo Adama University of Technology, Nigeria. \\ 4. Department of Biochemistry, Faculty of Basic Medical Sciences, Ladoke Akintola \\ University of Technology, Nigeria.
}

\begin{abstract}
The benefit of food as medicine is that healing and health promotion begins as soon as the next meal and this is known some decades ago as the significance of diet in health improvement, and prevention of diseases came to vanguard. Type 2 (non-insulin dependent) diabetes mellitus is a complex, multi-faceted disease particularized by high blood glucose (hyperglycemia), insulin resistance, pancreatic beta-cell dysfunction and altered insulin secretion. Recent data indicates a disturbing increase in the rate of hitches among individuals having type 2 diabetes mellitus despite the availability of several pharmacological involvements such as oral hypoglycemic agents and insulin therapy for its control. As a response to this, functional foods for example whole grains, cinnamon, legumes, nuts were developed, which are now vital substitutes for enhancing nutrition and public health. Therefore, investigation into the advantages of functional foods on type 2 diabetes mellitus is essential and can decide if these can be a proper substitute for the inhibiting, treatment and management of this pathology and related metabolic effects.
\end{abstract}

Keywords:- Functional Foods, Diabetes Mellitus, Insulin Therapy, Hyperglycemia, Bioactive Compounds, Antioxidants.

\section{INTRODUCTION}

The word diabetes mellitus (DM), basically called diabetes, depicts a metabolic dishevelment of numerous etiology represented by persistent high blood glucose with disorders in fat, protein and carbohydrate metabolism owing to abnormalities in insulin secretion/resistance. It has also been connected to severe long term difficulties, including retinopathy, nephropathies, neuropathies, myocardial infarctions, cerebral embolism and damage to the blood vessels.

Diabetes mellitus demonstrates a broad scope of distinction in incidence globally, being almost not present in New Guinea while diabetes mellitus notices part of Pima Indian populace (Pradeepa and Mohan 2002). In 1995, 135 million individuals were estimated to be diabetes by the international body world Health Organization (WHO) with a prediction to rise to approximately 300 million by 2025 (WHO 2008). In the milieu of the global epidemic of type 2 $\mathrm{DM}$ and obesity higher than $75 \%$ of the individuals are in the developing nations (Yoon et al., 2006). As regards the developed nations like the United states and Europe, the growing tendency is mostly as a result of the long-lasting survivors and the diabetics (Bruno and Landi 2011).

A novel relationship involving food and health in daily life has been shown by the specific health effects of functional foods (Henry 2010). Functional foods, among several definitions has been defined by the combined action of the European Commission on functional food science in Europe, as food produce with vital nutritional impact having beneficial effects on more than one role of the human organism, therefore enhancing the physical and general conditions likewise reducing the danger of emerging diseases. The volume of intake and variety of functional foods must be in consonance to its usual acceptance for nutritional purposes.

Consumers, however does not recognize functional foods as being therapeutics, however because they are concerned with health-associated matter, functional foods are then more likely to taken these functional foods possessing healthier benefits than the usual products (Sabbe et al., 2009). Over years, consumer requirement from the area of food production have been transformed significantly; they are aware that foods contribute immensely to their health (Mollet and Rowland 2002). At present, foods are not considered to satisfy hunger and to supply the required nutrients for humans only, however they can inhibit dietassociated pathologies, enhance mental and physical wellbeing of the consumers (Roberfroid, 2000). Increased costs of health-care services, high life expectations and objective for a quality life as contributed to the high demand of such foods.

There are three key abnormalities in the inception of hyperglycaemia in diabetes mellitus, which are high glucose production, decreased secretion and altered action of insulin (Chiasson \& Rabasa-Lboret 2004). Functional foods act systematically by boosting insulin secretion, promoting the absorption of glucose by the adipose tissues and skeletal muscle, preventing intestinal glucose absorption and production in the liver. To tackle diabetes epidemic, preventive approaches should note the complexity of disease progression, enhancing the natural ways for preventing, managing and treating the affected individuals, accurately prevailing in health claim food support, promotion and consumers' learning. Nowadays, numerous functional 
constituents are coming up due to their ability in prevention of diseases, while are broadly recognized and utilized as local medicine elements for decades.

The focus of this review is to discuss the promising function that functional foods has in the prevention, treatment and management of type 2 diabetes mellitus, with their wide capability with regards to cheap public health policies.

\section{FUNCTIONAL FOODS AND BIOACTIVE COMPOUNDS INVOLVEMENT IN THE TREATMENT OF TYPE 2 DM}

The idea of "functional foods" was conceived as a suitable and cost-effective resolution for persistent health challenges, being prominent in various branches of science. However, the denotation of "functional foods" varies among countries and cultures and has been defined and re-defined over decades. In addition to its nutritional potentials, functional foods significantly affect more than one function of the organisms by promoting the state of health, life quality and decrease the menace of diseases (Saito 2007). Hence two very important but different points in functional foods were integrated. Firstly, the science of nutrition, known for evaluating new compounds and developing foods and secondly, the industry known for its distribution and production of food which finally get to the consumers (Yamada et al., 2008).
Functional foods as fortified foods with special constituents with valuable physiological effects were first used in Japan. In order of significance, the foods had to fulfil nutritional needs such as constitute natural ingredients, be part of the diet on a daily basis and when taken, have a specific function in human, for example-

- Improve biological defense mechanisms

- Inhibition or recovery of several pathological conditions

- Maintain both the mental and physical condition

- Delay aging (Yamada et al., 2008).

The new speculation of functional foods as natural or processed foods having both essential or non-essential organically active constituents in precise quantity give a clinically verified and documented health advantage for the management, treatment or prevention of a chronic diseases was described by the Functional food centre (Martirosyan and Singh 2015). Presently, these functional foods are being developed with importance on (Martirosyan and Singh 2015):

1. Basic metabolic regulation: Metabolic efficiency includes glycemia optimization.

2. Antioxidant properties: Vitamin C, E, carotenoids, polyphenols of plant origin, has the potential to support the action of functional foods against oxidative attack.

3. Digestive system: probiotics, symbiotics and prebiotics, and are regarded as functional foods balancing the major flora in the intestine. Stability and array of the microbial gut microflora are significant in health maintenance.

4. Regulation of circulatory processes

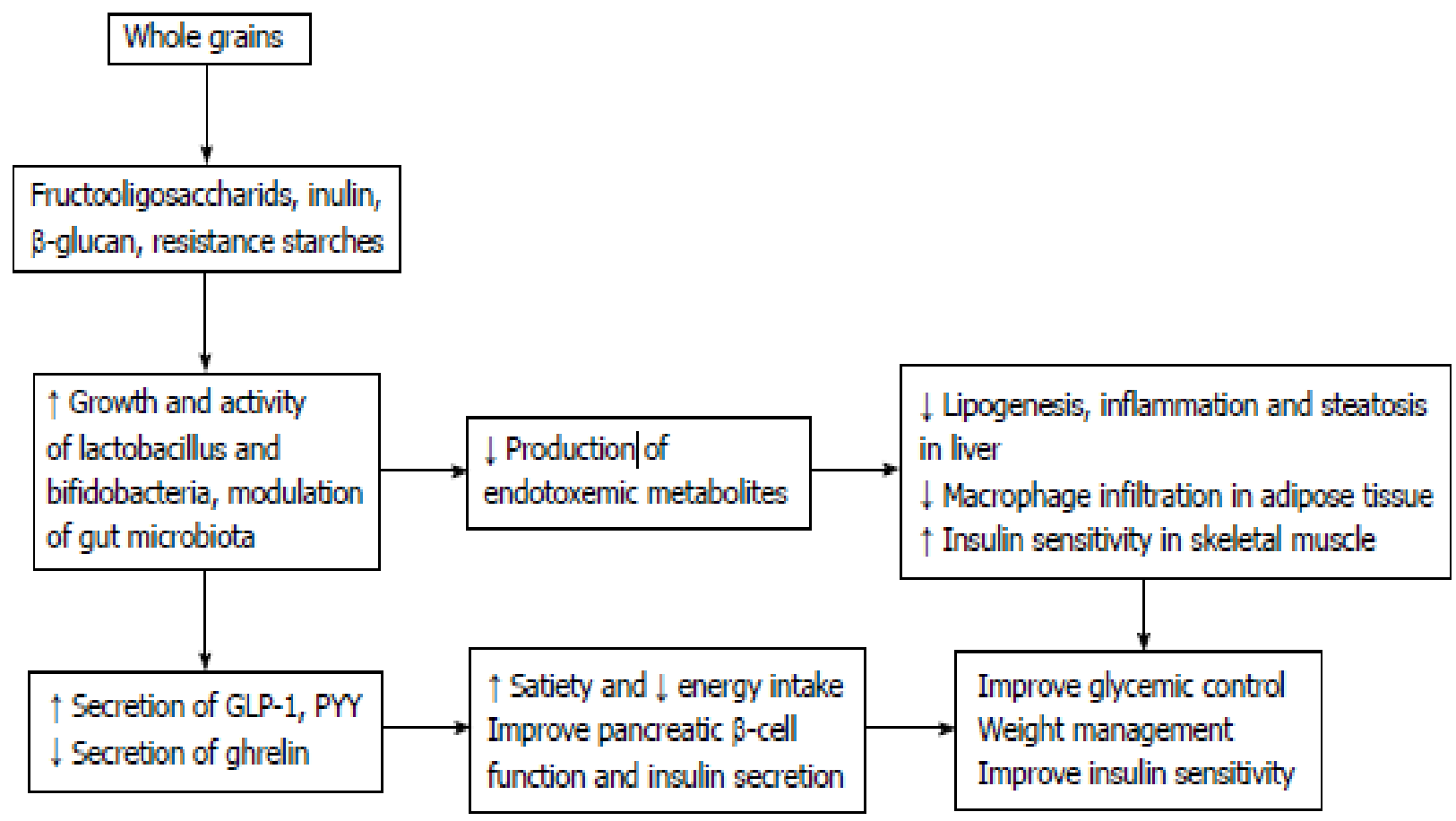

Fig. 1: Effect of Prebiotics constituents of Cereal-Based Products and Whole Grains in Glycemic Control 


\section{APPLICATIONS OF BIOACTIVE COMPOUNDS AND FUNCIONAL FOODS IN TYPE 2 DIABETES}

Presently, numerous bioactive compounds and functional foods with potential functions in the management or treatment of type 2 diabetes have been identified. Some of which include-

\section{Whole Grains}

The fundamental sources of energy supply and carbohydrates in human daily diets are the grains and cerealbased products. Utilization of functional grains such as cereals, whole grain, and bakery products fortified with oat, whole wheat, barley and rye is the primary measure in preparing a functional food-based diet because, the dietetic carbohydrate sources in type 2 diabetic individuals have been proved to have an influential role on glycemic index and insulin secretion. Past studies has shown that dietetic carbohydrate dysregulation in individuals with metabolic syndrome leads to favourable metabolic effects specifically on decreased adipocytes size, insulin sensitivity and altering adipocyte genes expression involved in insulin signaling (such as insulin receptors, hormone-sensitive lipase and insulin-like growth factor binding protein-5 (Laaksonen et al., 2005 and Kallio et al., 2007).

In contrast with refined grains, complex (nondigestible) polysaccharides like the $\beta$-glucan, soluble and insoluble inulin, fibers, and resistant starches, and also noncarbohydrate functional elements such as phytate, carotenoids, phytoesterogens, phenolic acids (caffeic acid, ferulic acid, vanilic acid) and tocopherols are more embedded in the whole grains.

Whole grain-based products with bioactive compounds has been shown to have protective effective against diabetes mellitus (non-insulin dependent), hypertension, metabolic syndrome, obesity, cardiovascular diseases, and different types of cancer (Borneo and León 2012). Glycemic index, increase insulin sensitivity, insulin secretion, pancreatic beta-cell effects could be effectively regulated with bioactive compounds of whole grains among other several mechanisms concerning the valuable effects of cereal-based products and whole grains in diabetic individuals (Shen et al., 2011). High levels of $\beta$-glucan, inulin and major soluble and fermentable fibers in whole grains, together with their hypoglycemic and hypolipidemic activities, act as prebiotics and regulate gut microbiota by enhancing growth and effect of lactic acid bacteria and bifidobacteria (Martínez et al., 2013), resulting to more metabolic responses (Fig. 1).

From the continuing evaluation of diabetes individuals, it was noticed that high intake of bran whole grains, fiber, cereals, and germs were related with reduce cardiovascular disease (CVD)-related death (He et al., 2010). Epidemiological investigations also established that regular intake of whole grains products can alter the major risk factors of atherosclerosis such as LDL-C and triglyceride levels, serum homocysteine levels, blood pressure and also vascular effects, inflammatory and oxidative status
(Anderson 2003). Also, oat meal products has been considered as healthy carbohydrate sources for diabetic cases; enrich with soluble fiber particularly antioxidants, beta-glucan and bioactive constituents such as phytate, carotenoid, phenolic acids (hydroxy-cinammic acids, ferulic acid, caffeic acid), phytosterols and flavonoids (Sadiq et al., 2008). Insulinemic lipidemic and glycemic responses in diabetic patients has been shown to be improved by increased consumption oat products and act as active constituent reducing post-prandial blood glucose level and inhibit pancreatic $\beta$-cell apoptosis as well (Tapola et al., 2005 and Bays et al., 2011).

High content of beta-glucan has been attributed to the favourable effects of barley and its by-products majorly in diabetes individuals; likewise in pre-diabetic patients, administration of barley $\beta$-glucan extract enhanced insulin resistance and index glucose tolerance (Shen et al., 2011); while the antioxidant, anti inflammatory activities and hypolipidemic properties of barley products have also been examined (Kofuji et al., 2012).

\section{$>$ Legumes}

Legumes such as beans, peas, peanuts, lentils are important sources of dietetic protein, non-digestible form carbohydrates such as oligosaccharides, resistance starches, dietary fiber and bioactive compounds like functional fatty acids (for example alpha-linolenic acid and linoleic acid), isoflavones (that is daidzein, genistein and glycitein), saponins, phenolic acids, phytate and some polyphenols (Duranti 2006). They are regarded as a constituent of healthy diet while evidence shows that regular intake of legumes has protective functions against type 2 diabetes mellitus, obesity, and cardiovascular disease (Flight et al., 2006); likewise, it can be considered as an essential constituent of a functional-foods based diet for the treatment or management of type $2 \mathrm{DM}$.

Bioactive compounds in beans and legumes such as $\alpha$ amylase, 7S globulin $\alpha$ chain and conglutin- $\gamma$ decreases the breakdown and absorption of dietetic carbohydrates, alter post-prandial glycemic response, maintain lipid and lipoprotein levels, regulate lipid metabolism (López-Amorós et al., 2006). Legumes are considered a functional food for diabetic individuals due to their high fiber, low glycemic index and phytochemical constituents. Beans can also be considered as an essential legume in the human diet having high level of omega-3 fatty acids, phytate, fiber, phenolic compounds and antioxidants. The hypoglycemic action of beans through the prevention of alpha-amylase and betaglucosidase activities has been shown to be related to antidiabetic drugs (Barrett et al., 2011; Yao et al., 2012).

Soybean enriched with distinct phytoesterogens (for example daidzein, genistein, glycitein), has been considered in diabetes; also the isoflavones and bioactive peptides of soybean have favourable actions on insulin sensitivity and glycemic control, kidney function and dyslipidemia (Anderson and Bush 2011). 
Soybean mainly interacts with estrogen receptors (ERs) to exert its anti-diabetic effects; previous studies have also shown that isoflavones in soy bind to both alpha and beta estrogen receptors selectively; ER- $\alpha$ is then attributed to the regulation insulin synthesis and secretion, pancreatic beta-cell survival and also modulate lipid and glucose metabolism as well (Gilbert and Liu 2013). Soy protein has been shown to enhance insulin sensitivity and enhance lipid homeostasis through peroxisome proliferator-activated receptor (PPAR) and liver $\mathrm{X}$ receptors activation and prevention of the sterol regulatory element binding protein1c. Constant intake of soy-products also helped in the management and/or treatment of dyslipidemia (Yang et al., 2011). Soy proteins and isoflavones has been confirmed to enhance biosynthesis of high-density lipoprotein cholesterol (HDL-C), induce low-density lipoprotein- cholesterol(LDLC) receptors, promote biosynthesis and excretion of bile acids, reduce steroids absorption in the gastro-intestinal (GI), induce beneficial effects in hormonal status such as the insulin- glucagon ratio, and thyroid hormones which results into enhancement of dyslipidemia (Borradaile et al., 2002).

\section{$>$ Nuts}

Apart from improving the general diet quality; currently, most evidence shows that intake of nuts has valuable effects on post-prandial glycemia after highcarbohydrate meals, reduces post-prandial oxidative stress, enhances insulin resistance, inflammatory processes, diminishes lipid atherogenicity, regulates lipid and lipoprotein levels (Li et al., 2009).

In addition, regular intake of nuts effectively aid in maintaining weight particularly in diabetic individuals. The anti-obesity potentials of nuts examined may be due to its stimulation of satiety, thermogenic effects, reduced nutritional fat absorption, and enhanced its excretion; bioactive constituents of nuts also alter adipose tissue metabolism, regulatory appetite neurotransmitters, and also reduces proliferation and differentiation of adipose tissues, promote fatty acid $\beta$-oxidation and prevent lipogenesis (Tey et al., 2011).

Furthermore, the valuable effect of nuts particularly in diabetic cases is favourably influential on high content of Larginine, which is the major precursor of nitric oxide; endothelial actions and also antioxidants and polyphenols may possibly contribute to this effect (Ros 2009). Diet enriched with nuts has been proven to be an efficient approach to control glycemic index and inhibit cardiovascular disease in type 2 diabetic individuals.

\section{Cinnamon}

Cinnamon is another functional- foods that has been assessed in depth as effective on diabetes mellitus. Broad review has confirmed that cinnamon has valuable effects on most factors related to antioxidants, glucose, insulin sensitivity, inflammation, lipids, blood pressure and body weight (Qin et al., 2010). Diminished insulin sensitivity is a pioneer to type $2 \mathrm{DM}$ and is always attributed to high fasting blood glucose (FBG) (Grant et al., 2009).

Anderson group (1990) were the first to report an in vitro study of insulin-promoting effects of cinnamon (Khan et al., 1990), and in addition characterized the bioactive constituents as water soluble polyphenols (Anderson et al., 2004). In human investigations, decreased the insulin receptors phosphorylation was observed in patients with type 2 DM (Cusi et al., 2000).

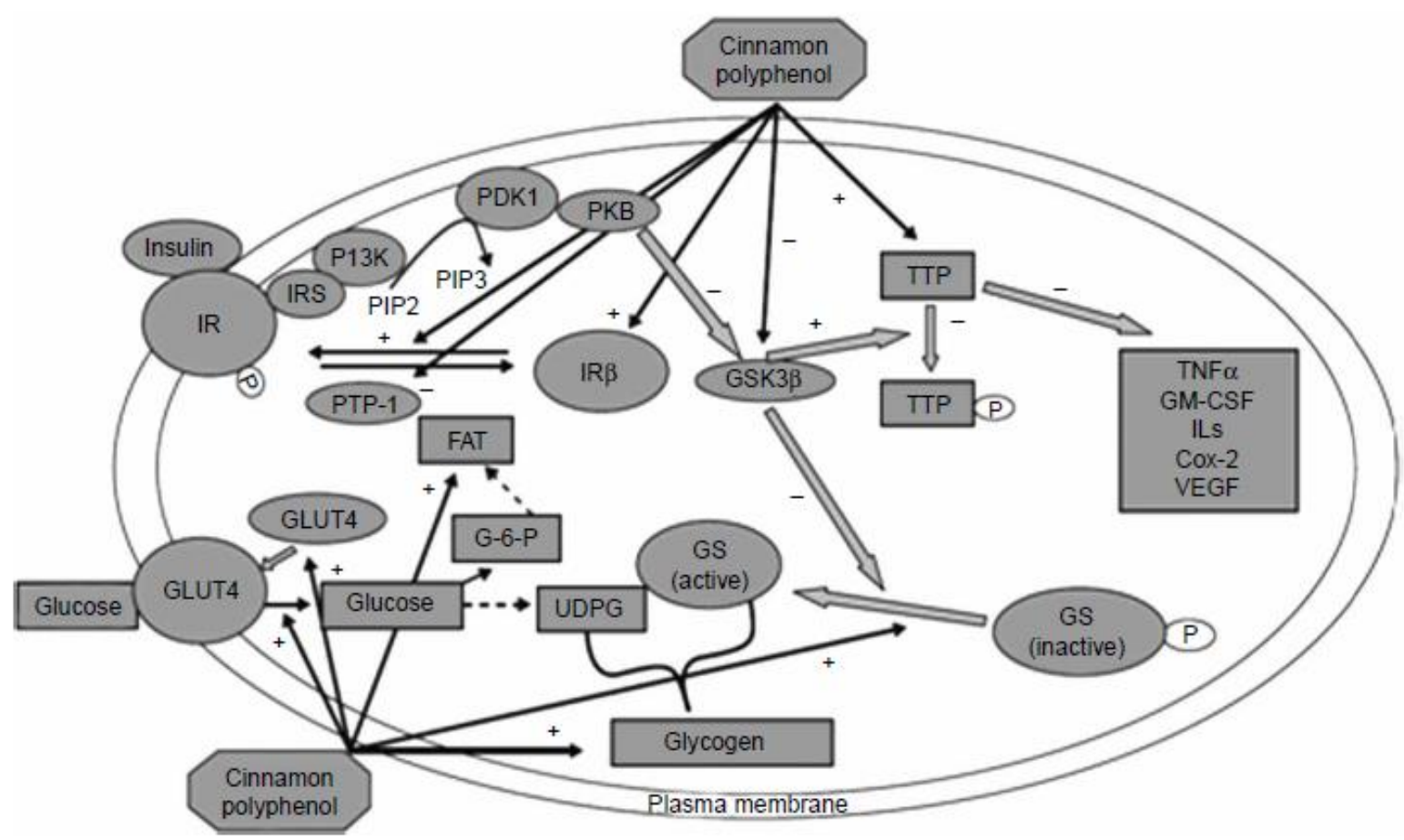

Fig 2- Activation of Insulin Receptor by Cinnamon Polyphenol (Pasupuleti and Anderson 2008) 
Of recent, Couturier et al (2011) study shows that addition of cinnamon to a diet-enhanced model of insulin resistant rat transformed insulin resistance and promoted liver glycogen in relation to the expression of several genes and proteins involving in glycogen metabolism and regulation of insulin sensitivity, although no noteworthy changes in the animals taking the cinnamon control diet. In 2003, Khan and colleagues study reports statistically and clinically important effects of cinnamon on lipid and glucose metabolism of type 2 DM- patients, numerous clinical trials were examined for the evaluation of cinnamon's potential to inhibit type $2 \mathrm{DM}$ or lessen the risk effects (Qin et al., 2010).

In vivo accumulation of advanced glycation end products (AGEs) involved in main pathogenic process in diabetics' complicacies has been proven to be significantly inhibited with the use of cinnamon bark. The inhibitory potentials were primarily characterized by the anti-glycation activity of a number of its phenolic constituents for example reactive carbonyl species entrapment (Peng et al., 2008).

\section{CONCLUSION}

Functional foods with their bioactive compounds including whole grains, legumes, nuts, cinnamons and so on, can regulate carbohydrate, lipid, lipoprotein and adipose tissue metabolism, hyperglycemia, insulin secretion and resistance, also enhance $\beta$ cell activities, weight management, regulate oxidative-antioxidative equilibrium, inflammatory processes and inhibit vascular ( both micro and macro) complications. Diet development based on these beneficial effects of healthy functional foods has been considered to be an efficient approach for management and treatment of diverse areas of diabetes and enhancement of health quality in diabetic individuals.

\section{REFERNCES}

[1]. MY. Donath (2013). Targeting inflammation in the treatment of type 2 diabetes. Diabetes, Obesity and Metabolism; 15(3):193-196

[2]. WHO (2012). Technical Report Series 894.

[3]. K. Yamada, Sato-Mito, Nagata J, K. Umegaki, (2008). Health Claims Evidence Requirements in Japan. Journal of Nutrition. 138:1192S-1198S

[4]. C.M. Hasler, A.C. Browm (2009). Association of American dietetic. Position of the American Dietetic Association- functional foods. Journal of the American Dietetic Association-109 (4 ):735-746

[5]. DM Martirosyan, J Singh (2015). functional food (A new definition) by FFC: Journal of functional foods in health and disease: 5 (6) : 209-223

[6]. DE Laaksonen, LK Toppinen, KS Juntunen, K Autio, KH Liukkonen, KS Poutanen, L Niskanen, Mykkänen H.M (2005). Dietary Carbohydrates Modification Enhances Insulin Secretion in patients with the metabolic syndrome. American Journal of Clinical Nutrition; 82: 1218-1227 [PMID: 16332654]
[7]. $\mathrm{P}$ Kallio, M Kolehmainen, DE Laaksonen, J Kekäläinen, T Salopuro, K Sivenius, L Pulkkinen, HM Mykkänen, L Niskanen, M Uusitupa, Poutanen K.S (2007). Dietary Carbohydrate Modification Induces Alterations in Gene Expression in Abdominal Subcutaneous Adipose Tissue in Patients with the metabolic syndrome: American Journal of Clinical Nutrition- 85: 1417- 1427 [PMID: 17490981]

[8]. R. Borneo, A.E. León (2012). whole- grains cerealsfunctional components and health benefits. Food Function; 3: 110-119 [PMID: 22134555 https://dx.doi. $10.1039 / \mathrm{c} 1$ fo10165j

[9]. RL Shen, FL Cai, JL Dong, XZ Hu (2011). Hypoglycemic effects and biochemical mechanisms of oat products on streptozotoc in induced diabetic mice. Journal of Agricultural Food Chemistry; 59: 88958900 DOI: $10.1021 / j f 200678 q$

[10]. I Martínez, JM Lattimer, KL Hubach, JA Case, J Yang, CG Weber, JA. Louk, DJ Rose, G Kyureghian, Peterson DA, Haub MD, Walter J. (2012). Gut Microbiome composition is related to whole grainsInduced Immunological Improvements. ISME Journal 2013; 7: 269-280 [PMID: 23038174 DOI: 10.1038/ismej..104]

[11]. M He, RM Van Dam, E Rimm, FB Hu, L Qi, (2010). Cereal Fiber, Whole- grain, Bran, and germ ingestion and the risks and CVD -specific mortality among women with type 2 DM. Circulation; 121: 2162-2168 [PMID: $20458012 \quad$ DOI: 10 1161/CIRCULATIONAHA. 109. 907360]

[12]. J.W. Anderson. (2003). Whole- grains guide against atherosclerotic CVD Proc Nutrition Society; 62: 135-142 [PMID: 12740068 DOI: 10.1079/PNS2002222]

[13]. M Sadiq Butt, M Tahir-Nadeem, MK Khan, R Shabir, MS Butt. (2008) Oat- unique among the cereals. European Journal Nutrition; 47: 68-79 [PMID: 18301937 DOI: 10.1007/s00394-008-0698 7]

[14]. N Tapola, H Karvonen, L Niskanen, M Mikola, E Sarkkinen. (2005). Glycemic Response of oat bran product in type 2 diabetic persons. Nutrition Metabolic Cardiovascular Disease; 15: 255-261- DOI: 10.1016/j.numecd.2004.09.003

[15]. H. Bays, J.L. Frestedt, M. Bell, C. Williams, L. Kolberg, W. Schmelzer, J.W. Anderson

[16]. (2011). Decreased viscosity barley beta-Glucan against placebo: a selected controlled trial of the effects on Insulin Sensitivity for people at risk of diabetes mellitus. Nutritional Metabolism (London); 8: 58 https://dx.doi.org 10.1186/1743-7075-8-58

[17]. M. Sakuma, H. Yamanaka- Okumura, Y. Naniwa, D. Matsumoto, M. Tsunematsu, $\mathrm{H}$ Yamamoto, $\mathrm{Y}$ Taketani, E Takeda (2009). Dose-dependent Effects of Barley cooked prepared whiterice on post-prandial glucose and desacyl-Ghrelin levels. Journal of Clinical Biochemical Nutrition; 44: 151-159- doi: 10.3164/ jcbn.08-232

[18]. K Kofuji, A Aoki, K Tsubaki, M Konishi, T Isobe, Y Murata (2012). Antioxidant Activity of beta- glucan. ISRN Pharmaceutical 2012;125864. 
[19]. D.A. Brockman, X. Chen, D.D. Gallaher (2013). Intake of a High beta- glucan barley flour improves glucose control and Fatty Liver and Increases Muscle Acylcarnitines in the zucker diabetic-fatty rat. European Journal of Nutrition; 52: 1743-1753 [PMID: 23229409]

[20]. M. Duranti (2006). Grain-legume proteins and nutraceutical properties. Fitoterapia; 77: 67-82 PMID: 16406359 doi: 10.1016/j.fitote.2005.11.008

[21]. Flight (2006) Clifton P. Cereal Grains and Legumes in the Prevention of Coronary Heart Disease and Stroke: a review of the literature. European Journal of Clinical Nutrition; 60: 1145-1159.

[22]. M.L. López-Amorós, T Hernández, I Estrella, (2006). Effect of germination on legume phenolic compounds and their antioxidant activity. J Food Comp Anal; 19 : 277-283 doi: 10.1016/j.jfca.2004.06.012

[23]. Barrett ML, Udani JK (2011). A proprietary $\alpha$-amylase Inhibitor from white-bean: A review of clinical studies on weight-loss and glycemic-control. Nutrional $J ; 10$ : 24 doi: 10.1186/1475-2891-10-24.

[24]. Y. Yao, X.Z. Cheng, L.X. Wang, S.H. Wang, G. Ren (2012). Major Phenolic Compounds, Antioxidantcapacity and Anti-diabetic potential of rice-bean (Vigna umbellata L) in China. International Journal Mol Sci; 13: 2707-2716-doi: 10.3390/ ijms13032707

[25]. SV Thompson, Winham DM, AM Hutchins (2012). Bean and rice meals reduce Post-prandial glycemicresponse in adults with type 2 diabetes: A Cross-over Study. Nutrional Journal; 11: 23- doi: 10.1186/14752891-11-23

[26]. J.W. Anderson, H.M. Bush. (2011). Soy-protein effects on serum Lipoproteins: A quality assessment and meta-analysis of randomized, Controlled Studies. Journal American Coll Nutrition; 30: 79-91 [PMID: 21730216 doi: 10.1080/07315724.2011.10719947]

[27]. B. Yang, Y Chen, T Xu, Y Yu, T Huang, X Hu, Li D. (2011). Systematic Review and Meta- Analysis of Soy-products Consumption in individuals with Type 2 Diabetes Mellitus. Asia Pacific Journal of Clinical Nutrition; 20: 593-602 [PMID: 22094845]

[28]. CF. Liu, Pan TM. (2011). Beneficial effects of bioactive peptides-derived from soybean on human health and their production by genetic engineering. Soy-bean and health: 311-329

[29]. CW Kendall, Esfahani A, J Truan, Srichaikul K, Jenkins DJ (2010). Health Benefits of Nuts in Prevention and Management of Diabetes. Asia Pacific Journal Clinical Nutrition- 19: 110-116 [PMID: 20199995]

[30]. TY. Li, AM. Brennan,. Wedick NM, C Mantzoros, Rifai N, FB Hu (2009). Regular Intake of Nuts is Related with a Lower Risk of Cardiovascular Disease in Women with Type 2 DM. Journal of Nutrition; 139: 1333-1338 -doi: 10.3945/jn.108.103622.

[31]. S.L. Tey., R. Brown, A. Gray, A Chisholm, C. Delahunty. (2011). Nuts Enhance Diet Quality Compared to other Energy Dense Snacks while Maintaining Body Weight. Journal of Nutritonal Metabolism; 357350.
[32]. E. Ros (2009). Nuts and Novel-Biomarkers of Cardiovascular Disease. American Journal of Clinical Nutrition; 89:1649S-1656S -doi:10.3945/ajcn..26736R

[33]. B. Qin, KS Panickar, Anderson RA (2010). Cinnamon: Its Potential Role in the Prevention of Insulin Resistance,Metabolic Syndrome and Type 2 Diabetes Mell. Journal of diabetes Science and Technology 4:685-693.

[34]. SJ Grant, Bensoussan A, Chang D, Kiat H, Klupp NL, Liu JP, Li X (2009). Chinese Traditional Medicines for Individuals with Impaired Glucose Tolerance/fasting blood glucose.

[35]. J Imparl-Radosevich, Deas S, Polansky MM,. Baedke DA, Ingebritsen TS, Anderson RA, Graves DJ (1998). Regulation of PTP-1 \& Insulin Receptor Kinase By Fractions From Cinnamon: Implications For Cinnamon Regulation of Insulin Signalling. Hormone Resistance 50:177-182

[36]. Khan, NA Bryden, M.M. Polansky, R.A. Anderson. (1990). Insulin Potentiating Factor \& Chromium Content of Selected Foods and Spices. Biological Trace Element Res 24:183-188.

[37]. K. Cusi, Maezono K, A. Osman, M. Pendergrass, ME. Patti, T. Pratipanawatr, (2000).

[38]. Insulin Resistance Differentially Affects the PI-3 kinase- and MAP-Kinase Mediated Signaling in Human Muscle. Journal of Clinical Investigation 105:311-320.

[39]. H. Cao, MM. Polansky, Anderson RA. (2007). Cinnamon-extract \& Polyphenols Affect the Expression of Tristetraprolin, Insulin-Receptor \& Glucose Transporter (GLUT) 4 in Mouse 3T3-L1 Adipocytes. Arch Biochemical Biophysics 459:214 222.

[40]. Couturier K, B Qin, Batandier C, M Awada, HiningerFavier I., F Canini et al. (2011).

[41]. Cinnamon Increases Hepatic Glycogen in an Animal Model of Insulin Resistance. Metabolism 60:1590 1597.

[42]. Khan A, M Safdar, MM. Ali-Khan, Khattak KN, RA. Anderson. (2003) Cinnamon Improves Glucose and Lipids of People with Type 2 Diabetes Mell. Diabetes Care 26:3215-3218.

[43]. Peng X, KW Cheng, Ma J, B Chen, Ho CT, Lo C (2008). Cinnamon-bark Pro-anthocyanidins as Reactive Carbonyl Scavengers to Prevent the Formation of Advanced Glycation End Products. Journal Agric Food Chemistry 56:1907-1911.

[44]. S. Lungarini, Aureli F, Coni E (2008). Coumarin and Cinnamaldehyde in Cinnamon Marketed in Italy: A Natural Chemical Hazard- Food Addition Contaminant Part A Chemical Analysis Control Exposure Risk Assessment 25:1297-1305.

[45]. NM Borradaile, de Dreu LE, Wilcox LJ, Edwards JY, Huff MW (2002). Soya Phyto estrogens, Genistein \& Daidzein, Decrease Apolipoprotein B Secretion from HepG2 Cells Through Multiple Mechanisms. Biochemical Journal; 366: 531-539 [PMID:12030847 doi: 10.1042/BJ20020046. 